\author{
Gopal Raman Sharma, MS \\ Department of Neurosurgery \\ National Academy of Medical Sciences \\ Bir Hospital, Kathmandu, Nepal \\ Prashant Kausal, MBBS \\ Department of Neurosurgery \\ National Academy of Medical Sciences \\ Bir Hospital, Kathmandu, Nepal
}

Rajiv Jha, MS

Department of Neurosurgery

National Academy of Medical Sciences

Bir Hospital, Kathmandu, Nepal

Nilam Khadka, MCh

Department of Neurosurgery

National Academy of Medical Sciences

Bir Hospital, Kathmandu, Nepal

Dhruba Raj Adhikari, MS

Department of Neurosurgery

National Academy of Medical Sciences

Bir Hospital, Kathmandu, Nepal

Prakash Bista, Mch

Department of Neurosurgery

National Academy of Medical Sciences

Bir Hospital, Kathmandu, Nepal

Pawan Kumar Sultania, MS

Department of Neurosurgery

National Academy of Medical Sciences

Bir Hospital, Kathmandu, Nepal

\author{
Address for Correspondence \\ Gopal Raman Sharma, MS \\ Department of Neurosurgery \\ National Academy of Medical Sciences \\ Bir Hospital, Kathmandu, Nepal \\ Email: gopal47@hotmail.com
}

Received, 11 December, 2011

Accepted, 21 December, 2011

\section{Outcome of Microsurgical Clipping of Intracranial Aneurysms in Bir Hospital}

Aneurysmal subarachnoid hemorrhage (SAH) is one of the serious and common neurosurgical problems in Nepal .In Nepal about 300000 people have intracranial aneurysms each year and out of them about 6000 people suffer from ruptured aneurysms. Data on the results of surgical management of intracranial aneurysms is very limited in Nepal. This is most probably the largest series of cerebral aneurysm surgery produced in Nepal till to date.

This is a retrospective study. There were 127 patients who harbored 152 intracranial aneurysms (ruptured and unruptured) and underwent microsurgical clipping over a period of five years (January 2007 to December 2011) at Bir Hospital, Kathmandu, Nepal There were 48(38\%) males and 79(62\%) females and age ranged from 21 to 80 years. Of them $81.9 \%$ had single and $18.1 \%$ had multiple aneurysms. Of 152,140 $(92 \%)$ were located in anterior and $12(8 \%)$ in posterior circulation. Most common locations were anterior communicating artery(33.7\%), middle cerebral artery bifurcation (26.8\%), internal carotid artery $(15.8 \%)$ and posterior communicating artery (10.6\%) respectively. Regarding size, $112(73.8 \%)$ aneurysms were $3-10 \mathrm{~mm}, 26(17.1 \%)$ were $10-25 \mathrm{~mm}$ and $13(8.5 \%)$ were $<3 \mathrm{~mm}$ and there was one giant aneurysm in our series. During surgery 13(10.2\%) aneurysms ruptured.

Good recovery after surgery was achieved in $84.4 \%$, moderately disabled in $7.8 \%$ and death in $7.8 \%$. Microsurgical clipping of intracranial aneurysms is the standard surgical treatment in developing countries where endovascular technique is not available or not affordable

Key words: cerebral aneurysms, microsurgical clipping, outcome
$\mathrm{P}$ revious literatures on natural history of cerebral aneurysms mentioned that about $2 \%$ of general population will have an intracranial aneurysms and an aneurysm will rupture in less than $1 \%$ of population and will be the cause of death in $0.5 \%$. ${ }^{30}$

About $89 \%$ of patients with intracranial aneurysms present with features of SAH and 7\% with local mass effect with or without haemorrhage. About $4 \%$ the aneurysms are are detected as an incidental. ${ }^{14}$
Features of warning leak preceding major aneurismal rupture have been reported in $20-59 \%$ of patients. They are usually attributed to aneurysm expansion or minor SAH. ${ }^{31}$

Clinical presentation remains a headache that is unusually severe for the patient and has a very sudden onset.

In $90-95 \%$ of the patients scanned within 24 hours of ictus demonstrates subarachnoid blood but becomes less 
effective with time, demonstrating subarachnoid blood in only $74 \%$ of patients scanned on $3^{\text {rd }}$ posthaemorrhage day and $50 \%$ of patients scanned on $7^{\text {th }}$ post haemorrhage day. ${ }^{2,28,29}$ If computed tomogram (CT) scan is negative and patient has classical clinical features of $\mathrm{SAH}$, a lumbar puncture and CSF analysis should be carried out .

Conventional cerebral angiogram or digital substraction angiogram (DSA) is still a gold standard diagnostic tool for the diagnosis of intracranial aneurysms. Multidimentional multislices MDMS CT angiogram is new emerging technique which is gradually replacing conventional angiogram. ${ }^{10,17}$ Magnetic resonance angiography (MRA) is other choice but less reliable and aneurysm diagnosis rate is less than DSA and computerized tomogram angiography (CTA).

Main goal of treatment of intracranial aneurysms is to prevent bleeding by occluding the neck of the aneurysms either by surgery or endovascular techniques. The surgical options are microsurgical clipping, wrapping, trapping, aneurysmorrhaphy and Hunterian ligation. . $^{30,31}$

On record the first planned intracranial operation for a saccular aneurysm was conducted by Norman Dott in 1933. ${ }^{11}$ He had wrapped the aneurysm with muscle fragment. The credit for the first definite treatment of a preoperatively diagnosed intracranial aneurysm goes to Dandy, who, in 1937 clipped the neck of an aneurysm with a metallic clip ${ }^{7}$.

Technical advances and general progress in microneurosurgery, neuro radiology, neuroanaesthesia and intensive care many neurosurgeons achieved progressively lower postoperative mortality rates after operation for intracranial aneurysms in 1960s and 1970s. Pre eminent among these workers are Yasargil ${ }^{18}$ and Drake ${ }^{12}$ who have established bench marks of excellence in huge series of anterior and posterior circulation aneurysms.

\section{Materials and Methods}

This is a retrospective study of 127 patients who presented with SAH harbored 152 intracranial aneurysms and underwent microsurgical clipping of aneurysms over a period of five years, from January 2007 to December 2011 at Department of Neurosurgery, National Academy of Medical Sciences, Bir Hospital, Kathmandu, Nepal. Those patients with intracranial aneurysms who underwent surgical procedures other than microsurgical clipping were excluded from this study. Initially all the patients with suspected aneurismal SAH had CT scan of brain and only few cases who had normal CT scan had undergone Lumbar puncture and cerebro spinal fluid (CSF) analysis. Diagnosis of intracranial aneurysms were made either by three vessels conventional cerebral angiogram or 64 slices MD CT angiogram. All the anterior circulation aneurysms were clippedvia pterional approach except pericallosal and A2 (Azygos) aneurysms which were clipped via frontal interhemispheric approach. Basilar artery (BA) tip and
BA-SCA (superior cerebellar artery) aneurysms were approached via subtemporal route whereas vertebral artery (VA) - posterior inferior cerebellar artery (PICA) aneurysms were approached either by far lateral suboccipital approach or midline posterior fossa craniectomy.Postoperatine plain CT scan of brain was carried out in all cases to rule out ischaemia, brain edema, haematoma and hydrocephalus. Postoperative 64 slices CT angiogram was performed in all cases prior to discharge to observe vasospasm, neck remnant and occlusion of other surrounding arteries. Outcomes were measured by GOS (Glassgow Outcome Score) and followed up in one week, one month, three months, six months and each year.

\section{Results}

There were 48 males and 79 females. Age ranged from 21 to 80 years and majority of patients belonged to the age group of 40 to 70 years.

On admission 6 patients had Hunt and Hess grade 1, 41 patients had grade 2, 59 patients had grade 3 and 21 patients had grade 4 and no patient had grade 5 in our series (Table 1).

All the patients with suspected aneurismal SAH on clinical ground were advised for cranial CT scan. On CT scan of brain 119 patients had intracranial haemorrhage and 8 patients had normal scan who were then subjected for lumbar puncture and CSF analysis to confirm the diagnosis of aneurismal SAH.

On CT scan findings according to Miller Fischer's grading $8.5 \%(9)$ had grade $0,5.7 \%(6)$ had grade I, $36.3 \%(44)$ had grade II, $29.5 \%(42)$ had grade III and 20\%(26) had grade IV respectively (Table 2 ).

Twenty three (18.1\%) patients had multiple (Figure 1) and $104(81.9 \%)$ patients had single aneurysms. Out of total $140(92 \%)$ aneurysms were located in anterior and $12(8 \%)$ were located in posterior circulation.

Forty seven $(33.7 \%)$ were anterior communicating artery aneurysms, 37 (26.8\%) were middle cerebral artery bifurcation aneurysms, 22(15.8\%) were internal carotid artery aneurysms and $15(10.6 \%)$ were posterior communicating artery aneurysms.

There were all together 12 aneurysms in posterior circulation and among them most common were basilar tip and PICA aneurysms (Figure 2). Size of aneurysm varied from $<3 \mathrm{~mm}$ to $>25 \mathrm{~mm}$ as shown in table (Table 3).

Twenty three (18.1\%) patients with cerebral aneurysms were operated within 72 hours and 81.9\% (104) were operated after one week of rupture. Thirteen (10.2\%) aneurysms ruptured during dissection of aneurysms. All the cases showed intact clips in situ in post operative cerebral angiography (Figure 3, 4).

Twenty six (20.5\%) patients suffered postoperative complications and among them most common complications were hydrocephalus(10), CSF leak with meningitis (4), 


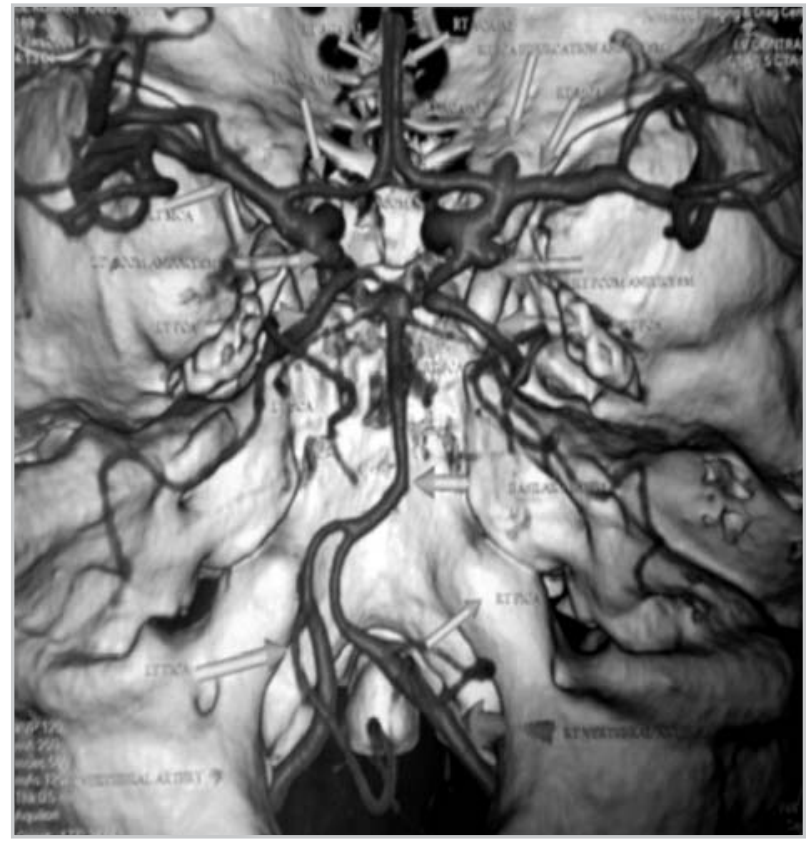

Figure 1. 64 Slices MD CT Angiogram showing Lt. Pcom., ICA bifurcation, MCA bifurcation aneurysms

hemiparesis(4), flap infection (2), third nerve palsy (2) (Table 4).

In our series good recovery was achieved in $107(84.4 \%), 10(7.8 \%)$ were severely disabled and death occurred in 10(7.8\%) (Table 5).

\section{Discussion}

Previous clinical studies have shown that $60 \%$ of these patients with aneurismal SAH die or severely disabled ${ }^{6,20}$ and remaining patients one half have significant neurophysiological and cognitive deficit and are unable to return to work. The primary cause of death and disability are the effect of the initial hemorrhage, subsequent rebleeding and vasospasm.

After the aneurysmal SAH about 20 to $30 \%$ of ruptured aneurysms rebleed within 30 days and then at a rate of approximately $3 \%$ per year and more than $70 \%$ of patients who rebleed die. . $31^{3}$

Rebleeding of the aneurysms can be prevented only by obliterating the aneurysm by direct surgery or endovascular technique. ${ }^{31}$

The dawn of modern cerebral aneurysm surgery came in 1933 when Egas and Moniz demonstrated an intracranial aneurysms by the cerebral angiography which they had discovered. ${ }^{30}$

Norman Dott in 1933 performed first aneurysm surgery ${ }^{11}$ by stuffing a muscle fragment against an aneurysm that had ruptured intraoperatively and succeeded in stopping bleeding and obtaining a good long term result.

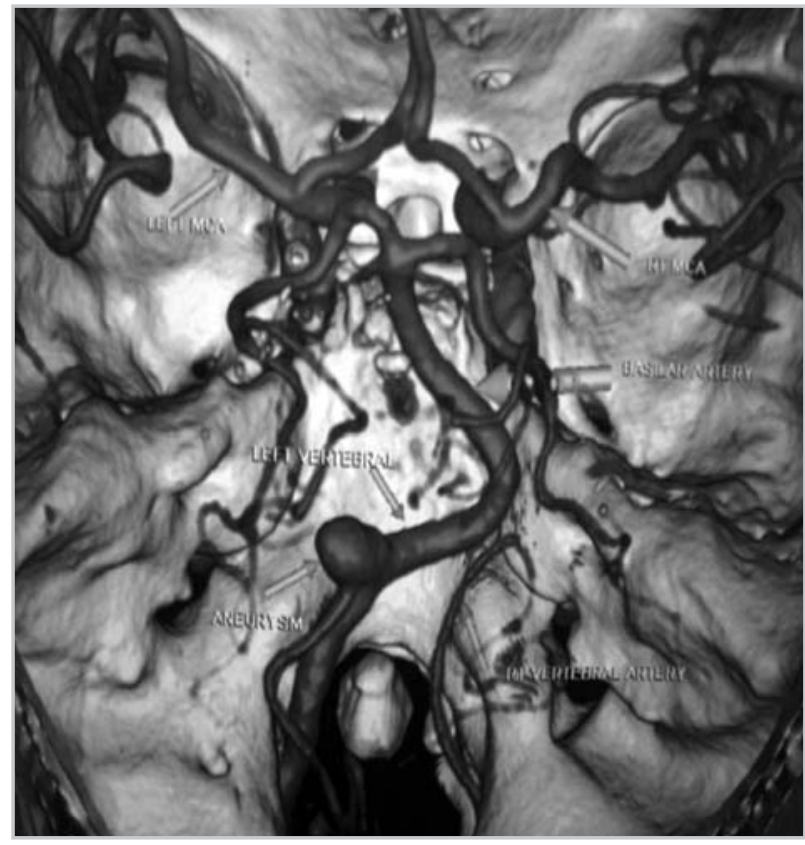

Figure 2. Cerebral CTA depicting large VA-PICA aneurysm

Walter Dandy carried out the first definitive treatment of a preoperatively diagnosed intracranial aneurysm by clipped the neck of an aneurysm with a metallic clip.

Over a period of five years, from January 2007 to December 2011, 127 patients harboring 152 intracranial aneurysms undergone microsurgical clipping under general anesthesia in our institute.

There were $48(38 \%)$ male and $79(62 \%)$ female in this retrospective study and these findings are similar to literatures that cerebral aneurysms are more common in female than male ${ }^{30,31}$, however, reason behind this is not known.

Intracranial aneurysm is a neurosurgical problem of middle and old age people and rarely occurs in children and young adults. The incidence of aneurismal rupture is gradually increases for each decade and peaks in $6^{\text {th }}$ decade. ${ }^{30,31}$ Our series also shows the similar result that majority of patients belonged to 40-70 age groups.

In this study CT Scan of brain was carried out in all suspected cases of aneurismal SAH . Out of 127 cases 9 patients had normal CT Scan for which they undergone Lumbar puncture and CSF analysis to confirm the diagnosis. $85 \%$ aneurismal SAH were diagnosed by CT Scan of brain and remaining $15 \%$ were diagnosed by lumbar puncture in this series. This result is supported by the results of previous studies Which have revealed that CT Scan demonstrates subarachnoid blood in $90-95 \%$ of patients scanned within 24 hours of haemorrhage but becomes less effective with time, demonstrating subarachnoid blood in only $74 \%$ of patients scanned on the third posthaemorrhage 


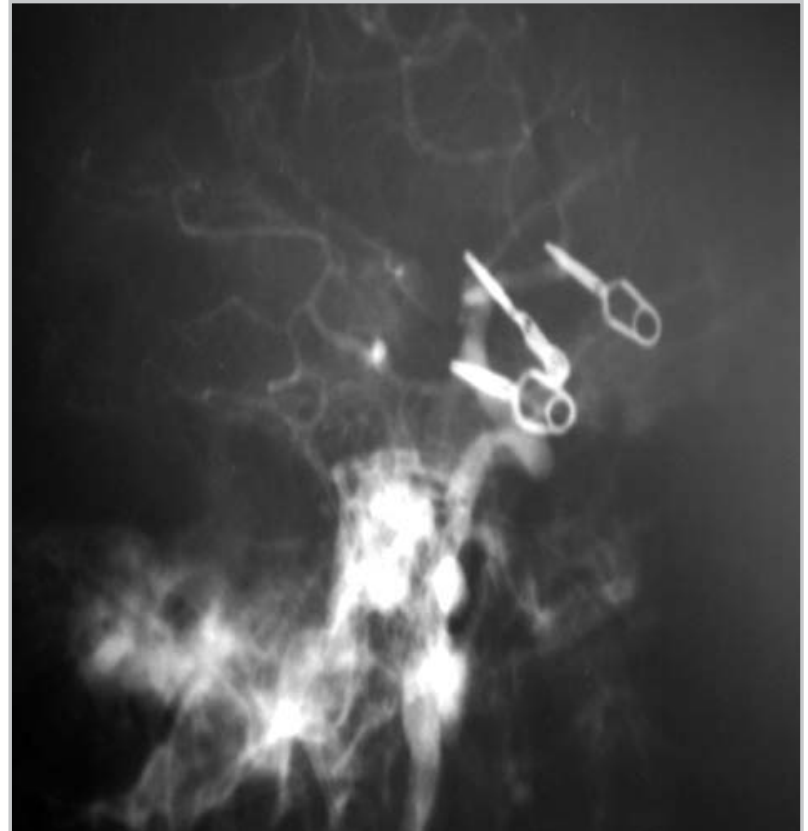

Figure 3. Postoperative Cerebral conventional angiogram showing total occlusion of necks of all aneurysms and clips in situ.

day and $50 \%$ of the patients scanned on the $7^{\text {th }}$ posthaemorrhage day. ${ }^{2,28,29}$ If CT is negative, a lumbar puncture should be carried out.

Our study reveals that out of 127 cases of ruptured aneurysms $75.6 \%$ (96) had SAH, 19\%(25) had ICH, $11 \%(20)$ had Intraventricular haemorrhage and 16.5\%(15) had hydrocephalus on CT Scan of brain and these CT findings are similar with the CT findings of published series. ${ }^{5,22}$ Aneurysms arising from the distal anterior cerebral arteries are the most likely to produce intracerebral haematoma, however, relatively rare. Intracerebral haematomas are more commonly seen with aneurysms of MCA and Acom.artery. Intracerebral haematomas complicated $34 \%$ of aneurysm cases reported by Pasqualin. ${ }^{23}$. In our series most common aneurysms causing intracerebral haematomas were MCA, Pericallosal and ACom.artery aneurysms respectively.

Intraventricular haemorrhage complicates an aneurysms rupture in $13 \%$ to $28 \%$ of clinical series and $37 \%$ to $54 \%$ of autopsy series. The anterior communicating artery, basilar tip artery aneurysms are the most common to cause intraventricular haemorrhage. ${ }^{22,24}$ Over $50 \%$ of patients with large intraventricular haemorrhages are admitted to poor grades, and the mortality in such cases exceeds $64 \%$. ${ }^{22}$ In our series most common causes of intraventricular haemorrhage were A Com. Artery, basilar tip and PICA aneurysms.

Hydrocephalus was observed in $16.5 \%$ (15) of patients with aneurismal SAH which is comparable with Sheehan's series which was about $20 \%$. ${ }^{24}$

Conventional cerebral angiogram or DSA is gold

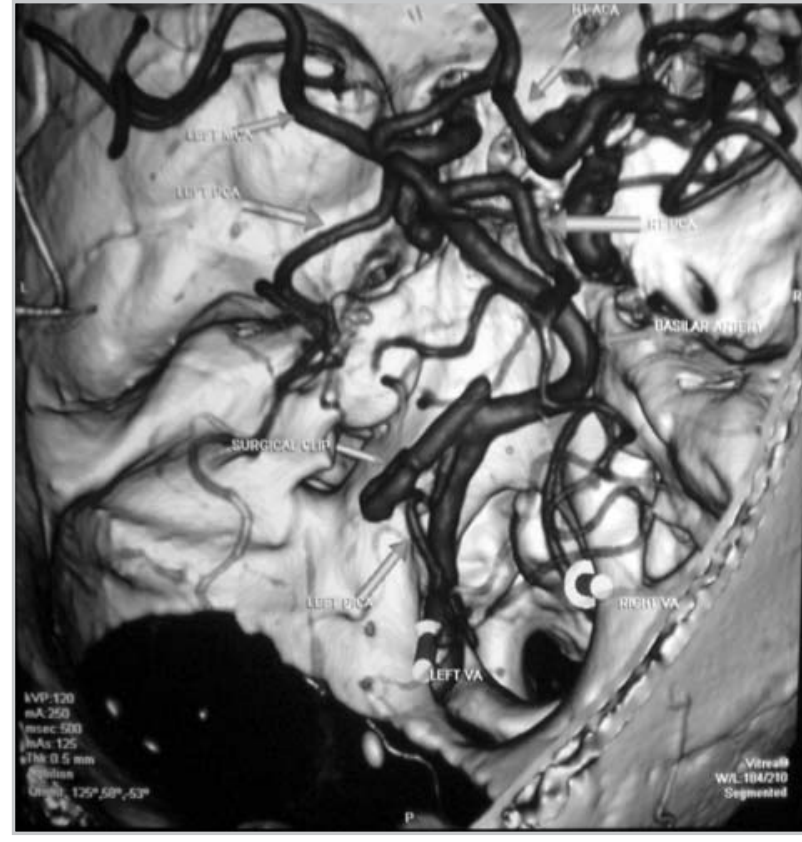

Figure 4.Postoperative cerebral CTA showed total occlusion of VA-PICA aneurysm and clip in proper position.

standard for the evaluation of cerebral aneurysms and demonstrates source in $80-85 \% .{ }^{15}$ The role of CTA is just now becoming defined but appears to hold promise, with a reported sensitivity of $95 \%$ and specificity of $83 \%$ in detecting aneurysms as small as $2.2 \mathrm{~mm}$. Unlike conventional angiography, CTA shows a 3-D image and demonstrate the relation to near by bony structure. ${ }^{10,17}$ If we want to study cross flow and occlusion test of cerebral circulation then conventional angiogram is mandatory.

In the beginning of the study we did conventional crerebral angiogram in all cases of aneurismal SAH but later on after the availability of CTA in town we have been advising CTA in all cases of suspected cerebral aneurysms because it is minimally invasive, safe, cheap, quick, available as an emergency service and aneurysm detection rate is as high as conventional angiogram or DSA (Figure 1 and 2).

In our series $18.1 \%$ (23) were multiple and $81.9 \%$ (104) single aneurysms and Wirth ${ }^{32}$ has shown similar result and in his study multiple aneurysms were present in $15-33.5 \%$ of cases of SAH. In suzuki's personal series of 1080 cases single aneurysm constituted $85 \%$ and multiple aneurysms in $15 \% .{ }^{25}$

In our study $92 \%$ (140) patients had anterior and $8 \%(12)$ posterior circulation aneurysms which is similar to the findings of other published intracranial aneurysm series. ${ }^{16,30}$

Among anterior circulation aneurysms most common location in our series were Acom.artery,middle cerebral artery bifurcation, Internal carotid artery and PCom.artery whereas in others series most common location were 
Acom.artery, Pcom.artery, middle cerebral artery and internal carotid artery. These differences might be due to small number of patients in our study and probably it is to early to draw the final conclusion.

In posterior circulation, most common location were basilar tip and PICA which matches the results of international literature on intracranial aneurysms. ${ }^{30,31}$

In this series, $73.8 \%(112)$ aneurysms were $3-10 \mathrm{~mm}$ in size, $17.1 \%(26)$ were $10-25 \mathrm{~mm}$ and $8.5 \%(13)$ aneurysms were less than $3 \mathrm{~mm}$ in size. There was one giant aneurysm in our series (Table 4).Previous experiences have shown that about $5 \%$ of intracranial aneurysms are giant. ${ }^{30}$ This is too early to comment on it and we may receive giant aneurysms in future when we will have more number of patients.

It is our protocol in institute that if a patient with aneurismal SAH comes within 72 hours of ictus we do emergent cerebral angiogram and clipping of aneurysms. Hence, $18.1 \%$ (23) were operated within 72 hours of ictus as an emergency procedure and $104(81.9 \%)$ were operated after one week of rupture. We usually do not operate the patients in 3-7 days of ictus because this is the period of vasospasm and postoperative complications rate would be very high if we clip the aneurysms during these period. ${ }^{26}$

Intraoperatively aneurysm ruptured in $10.2 \%(13)$ and ruptured mostly occurred during dissection of aneurysm sac. In Batjer and et al series intraoperative rupture occurred in $19 \%{ }^{4}$ The cooperative study dealing with 228 patients randomized to surgical treatment between June 1963 and Feb 1970 reported a surprisingly low $18 \%$ incidence of intraoperative aneurysm rupture, presumably without the benefit of microsurgical clipping. ${ }^{16}$ Literature reviews have shown that majority of intraoperative aneurysms ruptures in the subarachnoid space prior to clip.

Twenty six $(20.5 \%)$ patients developed surgery related postoperative complications after the microsurgical clipping of aneurysms. 10 patients underwent VP Shunt for hydrocephalus. 4 patients had developed postoperative CSF leak followed by meningitis which were treated by wound repair and a course of intravenous antibiotics. 2 patients got flap infection which were managed by flap removal and intravenous antibiotics. 2 patients had transient ipsilateral $3^{\text {rd }}$ nerve palsies who underwent microsurgical clipping of basilar tip aneurysms via subtemporal approach.

Hemiparesis was developed in 5 patients due to vasospasm followed by cerebral ischaemia. Postoperative Complications in our series were similar to complications shown in other previously published series. ${ }^{30}$

Good recovery was achieved in $84.4 \%$ (107), $7.8 \%$ (10) were severely disabled and 7.8\%(10) died of surgery related complications in our series of 127 cases. Mortality rate is 4\% in Yasargil's series ${ }^{18}, 5 \%$ in Suzuki's series ${ }^{25}$, $6 \%$ in Sundt' series ${ }^{26}$ and $7 \%$ in Weir and Aronyk' series. ${ }^{29}$ In Tang and et al series of 120 cases, good recovery was achieved in $94(78.4 \%)$ cases, 5(4.2\%) remained severely disabled and $2(1.6 \%)$ died. ${ }^{27}$ In Devkota's series of 40 patients, good recovery was made in $90 \%$ and $10 \%$ died and there were no severely disabled patients in his study groups. ${ }^{9}$ Over all surgical outcome is favorably comparable with other published series despite our resource constraint.

We routinely do CT scan of brain on $1^{\text {st }}$ postoperative day in all cases after microsurgical clipping of cerebral aneurysms to see edema, ischaemia, haematoma and hydrocephalus.Prior to discharge CT angiogram is performed to observe unexpected findings like aneurismal rest, unclipped aneurysm or major vessel occlusion. So far, in our series we have not found aneurismal rest, partial occlusion of aneurysms and major vessel occlusion on postoperative CTA ( Figure 2 and 4 ).

Literature also suggests routine postoperative angiography after microsurgical clipping of intracranial aneurysms. ${ }^{15,21}$

Over all more than $90 \%$ of aneurysms underlying surgical clip occlusion are completely obliterated at surgery ${ }^{1,8}$, however, vessel occlusion or aneurysms remnants may be found on $5 \%$ of postoperative angiogram. ${ }^{13,19}$

\section{Conclussions}

Microsurgical clipping is the standard surgical treatment for intracranial aneurysms and our results are as good as the results of other developed countries. This surgical treatment remains the best treatment for intracranial aneurysms in developing countries like Nepal where endovascular technique is not available or financially not viable at present economic scenario of our country.

\section{References}

1. Acevedo JC, Turjman F, Sindou M. Postoperative angiography in surgery for intracranial aneurysm: prospective study in consecutive series of 267 operated cases. Neurochirgie 43: 273-284, 1997

2. Adams HP Jr, Kassell, Torner JC, et al. CT and clinical correlation in recent aneurismal subarachnoid haemorrhage: a preliminary report of the cooperative aneurysm study. Neurology 33: 981-988, 1998

3. Alvord EC, Loeser JD, Barley WL, et al. Subarachnoid haemorrhage due to ruptured aneurysms:A simple method of estimating prognosis. Acta Neurol 27: 273-284,1972

4. Batjer H, Samson D. Intraoperative aneurismal rupture : Incidence, outcome, and suggestions for surgical management. Neurosurgery 18: 701-707, 1986

5. Benoit RG, Cochrane DD, Durity F et al. Clinical radiological correlates in intracerebral haematomas due to aneurismal rupture. Can J Neurol Sci 9: 409-414, 1982

6. Broderic JP, Jhoma GB, Dudler JE, et al. Initial and recurrent bleeding are the major causes of death following subarachnoid haemorrhage. Stroke 25: 
1342-1347, 1994

7. Dandy WE. Intracranial aneurysms of the internal carotid artery cured by operation. Ann Surg 107: 654-659, 1938

8. David CA, Vishteh G, Spetzler RF, et al. Late angiographic follow up of surgically treated aneurysms. J Neurosurg 91: 396-401,1999

9. Devkota UP, Aryal KR. Result of surgery for ruptured intracranial aneurysms in Nepal. Br J Neurosurg 15: 13-16,2001

10. Dorsch NWC, Young N, Kingston RJ, et al. Early experience with spiral CT in the diagnosis of intracranial aneurysms. Neurosurgery 36: 230238, 1995

11. Dott NM. Intracranial aneurysms: Cerebral arterioradiography: surgical treatment. Edinburg Med J 40: 219-240,1933

12. Drake CG. Management of cerebral aneurysms. Stroke 12: 273-283, 1981

13. Drake CG, Friedman AH, Peerless SJ. Failed aneurysm surgery: reoperation in 115 cases. J Neurosurg 61: 848-856, 1984

14. Fox JL. Intracranial aneurysms.New York: Springerverlag. 1983

15. Gerardin E, Tollard E, Derrey S, et al. Usefulness of multislice computerized tomographic angiography in the postoperative evaluation of patients with clipped aneurysms. Acta Neurochir (wien) 152: 793-802, 2010

16. Graf CJ, Nibbelink DW. Randomized treatment study: intracranial surgery. In Sahs AL. Nibbelink DW, Torner JC (eds): Aneurysmal subarachnoid haemorrhage: Report of the cooperative study. Baltimore:Urban and Schwarzenburg:145-202, 1981

17. Hsiang JNK, Liang EY, Lam JMK, et al. The role of computed tomographic angiography in the diagnosis of intracranial aneurysms and emergent aneurysm clipping. Neurosurgery 38: 481-487, 1996

18. Krayenbuhl HA, Yasargil MG, Flamm ES, et al. Microsurgical clipping of intracranial saccular aneurysms. J Neurosurg 37: 1-6, 1972

19. Le Roux P, Elliott JP, Eskridge JM, et al. Risks and benefits of diagnostic angiography following aneurysm surgery: A retrospective analysis of 597 studies. Neurosurgery 42: 1248-1255

20. Ljunggren B, Saveland H, Brandt L, et al . Aneurysmal subrachnoid haemorrhage: Total annual outcome in a 1.46 million population. Surg Neurol 22: 435-438, 1984

21. Macdonald RL, Wallace C, Kestle JRW. Role of angiography following aneurysm surgery. J Neurosurg 79: 826-32, 1993

22. Mohr G, Ferguson G, Khan M, et al .Intraventricular haemorrhage from ruptured aneurysm: Retrospective analysis of 91 cases. J Neurosurg 58: 482-487, 1983

23. Pasqualin A, Bazzan a, Cavazzani $P$, et al. Intracranial haematomas following aneurismal rupture: Experience with 309 cases. Surg Neurol 25: 6-17, 1988

24. Sheehan JB, Polin RS, Sheehan JM, et al. Factors associated with hydrocephalus after aneurismal subarachnoid haemorrhage. Neurosurgery 45: 1120-1128, 1999

25. Sujuki J. Multiple aneurysms: treatment. In Pia HW, Longmaid C, Zierski J (eds): Cerebral aneurysms: Advances in diagnosis and therapy. Berlin : Springer, pp 352-363,1979

26. Sundt TM Jr. Cerebral vasospasm following subarachnoid haemorrhage: evolution, management, and relationship to timing of surgery. Clin Neurosurg 24: 228-247, 1977

27. Tang W, Feng H, Chen Z, et al. Mirosurgical treatment of ruptured intracranial aneurysms: a 120 case analysis. Acta Neurochir suppl 110: 141-143, 2011

28. Van Gijn J ,Van Dongen KJ. The time course of aneurismal haemorrhage on computed tomograms. Neuroradiology 23: 153-156, 1982

29. Weir B, Aronyk K. Management and postoperative mortality related to timing of clipping for supratentorial aneurysms: a personal series. Acta Neurochir (Wien) 63: 135-139, 1982

30. Weir B, Macdonald RL. Intracranial aneurysms and subarachnoid haemorrhage:An overview. In Wilkins RH, Rengachary SS (eds): Neurosurgery. 2 ed .Vol 2. Mc-Graw-hill, New York, pp 2191-2213,199

31. Winn HR, Richardson AE, Jane JA. The long term prognosis in untreated cerebral aneurysms 1 . The incidence of late haemorrhage in cerebral aneurysms: A 10 year evaluation of 364 patients. Ann Neurol 1: 358-370, 1977

32. Wirth FP. Surgical treatment of incidental intracranial aneurysms. Clin Neurosurg 33: 125-135, 1986 